\title{
Determinants of preeclampsia in women with type 1 diabetes
}

\author{
Paweł Gutaj $^{1}$ - Agnieszka Zawiejska ${ }^{1}$ - Urszula Mantaj ${ }^{1}$ Ewa Wender-Ożegowska ${ }^{1}$
}

Received: 8 July 2017 / Accepted: 8 September 2017 / Published online: 3 October 2017

(C) The Author(s) 2017. This article is an open access publication

\begin{abstract}
Aims Despite improvement in diabetic care over the years, the incidence of hypertensive disorders of pregnancy is still very high. Therefore, the aim of our study was to determine risk factors for PE in women with T1DM.

Methods This study was a prospective, nested case-control study on a population of 165 women with T1DM. Women were divided into 3 subgroups: normotensive $(N=141)$, gestational hypertension $(\mathrm{GH})(N=8)$, and $\mathrm{PE}(N=16)$. Clinical data were collected in the first trimester ( $<12$ th week), in mid-pregnancy (20-24th weeks), and just prior to delivery (34-39th weeks). IR in the first trimester was quantified using the estimated glucose disposal rate formula (eGDR, milligrams/kilogram/minute). Simple logistic regression was used to search for factors associated with PE and GH. For multivariate comparisons, we used multiple logistic regression with stepwise selection.

Results All preeclampsia cases were diagnosed in primiparae. The presence of vasculopathy was the strongest determinant of PE (OR 10.8, 95\% CI 3.27-35.97, $P=0.0001$ ), followed by a history of chronic hypertension $(6.05,1.75-20.8, P=0.004)$ and the duration of diabetes $(1.11,1.03-1.12, P=0.009)$. However, chronic hypertension and duration of diabetes were no longer associated with PE after adjustment for the presence of vasculopathy. Higher gestational weight gain (GWG) was associated with PE, and this association remained significant after
\end{abstract}

Managed by Massimo Porta.

Paweł Gutaj

pgutaj@o2.pl

1 Division of Reproduction, Department of Obstetrics, Gynecology and Gynecological Oncology, Poznan University of Medical Sciences, 33 Polna St, 60-535 Poznań, Poland adjustment for first trimester body mass index $(1.14,1.02-1.28$, $P=0.02$ ). Both systolic and diastolic blood pressure assessed in the first trimester were significant determinants of PE; however, this association was no longer observed after adjustment for the presence of chronic hypertension. Glycated hemoglobin $\left(\mathrm{HbA}_{1 \mathrm{c}}\right)$ levels from all 3 trimesters were significantly associated with PE (first trimester: 1.38, 1.01-1.87, $P=0.04$; second trimester: $2.76,1.43-5.31, P=0.002$; third trimester: $2.42,1.30-4.51, P=0.005)$. There was a negative association between eGDR and PE $(0.66,0.50-0.87, P=0.003)$. Among lipids, triglycerides (TG) in all 3 trimesters were positively associated with $\mathrm{PE}$, and this association was independent of $\mathrm{HbA}_{1 \mathrm{c}}$ levels (first trimester: 5.32, 1.65-17.18, $P=0.005$; second trimester: $2.52,1.02-6.26, P=0.05$; third trimester: 2.28 , $1.39-3.74, P=0.001$. We did not find any predictors of $\mathrm{GH}$ in the regression analysis among all analyzed factors.

Conclusions Primiparity and diabetic vasculopathy seem to be the strongest risk factors for PE in women with type 1 diabetes. However, preexisting hypertension and higher GWG were also associated with PE in women with T1DM. Among laboratory results, higher $\mathrm{HbA}_{1 \mathrm{c}}$ and TG levels in all 3 trimesters were associated with PE. The association between higher IR and PE in women with T1DM needs further study.

Keywords Pregestational diabetes - Type 1 diabetes . Preeclampsia $\cdot$ Gestational hypertension

\section{Introduction}

Preeclampsia (PE) is a leading cause of maternal and neonatal morbidity and mortality. It complicates around 3\% of pregnancies. There are several risk factors for PE including primiparity, multifetal gestation, maternal age, and pregestational 
diabetes [1]. The incidence of PE among diabetics ranges from 10 to $20 \%$. Among women with diabetic nephropathy, these numbers are much higher [2]. The pathogenesis of PE is still not fully understood, but it seems to be a disease of placental origin. A diabetic environment as well as preexisting maternal vasculopathy can predispose to reactive oxygen species formation and affect placental function from early pregnancy [3]. Hyperglycemia has been also found to be associated with PE. However, there are some discrepancies between studies regarding what time of action of hyperglycemia is crucial for the development of PE. Temple et al. [4] showed that both preconceptional counseling and first trimester glycated hemoglobin $\left(\mathrm{HbA}_{1 \mathrm{c}}\right)$ were not associated with PE. Elevated levels of total cholesterol, non-high-density lipoprotein (non-HDL) cholesterol, and triglycerides (TG) during all trimesters of pregnancy, as well as lower levels of high-density lipoprotein (HDL) cholesterol in the third trimester, were associated with PE; however, data from the diabetic population are scarce [5]. The same refers to insulin resistance (IR) and other components of metabolic syndrome [6]. The aim of this study was to establish risk factors for PE in a prospectively recruited cohort of women with type 1 diabetes (T1DM) managed by a single tertiary obstetric center.

\section{Materials and methods}

This prospective study was conducted in the Gynecologic and Obstetrical University Hospital in Poznan, Poland, in a group of 165 pregnant women with T1DM during a period between June 2012 and December 2014. Anthropometric, clinical, and laboratory data were collected during 3 standard hospitalizations that took place in our hospital in the first trimester $(<12$ th week of gestation-I), in mid-pregnancy (20-24th weeks of gestation-II), and just prior to delivery (34-39th weeks of gestation-III). A detailed description of the study protocol was previously described elsewhere $[7,8]$. The therapeutic target in women diagnosed with hypertensive disorders of pregnancy was blood pressure $<135 / 85 \mathrm{mmHg}$ [9].

Women were divided into 3 subgroups according to pregnancy outcome: normotensive $(N=141)$, developing gestational hypertension $(\mathrm{GH})(N=8)$, and developing PE $(N=16)$.

Blood samples were taken after overnight fasting and immediately transported to the central laboratory of the Gynecologic Obstetrical University Hospital in Poznan for analysis. $\mathrm{HbA}_{1 \mathrm{c}}$ level in whole blood was determined using the turbidimetric inhibition immunoassay (TINIA) (Tina-quant Hemoglobin A1c II test in a Cobas c311 analyzer [Roche Diagnostics, Basel, Switzerland]). The normal range for this test is $4.8-6.0 \%$ (29-42 $\mathrm{mmol} / \mathrm{mol})$ for a nonpregnant population.
Total serum cholesterol, HDL cholesterol, and TG levels were determined with Roche Diagnostics reagents (Cholesterol CHOD-PAP, HDL-C plus, and Triglycerides GPOPAP, respectively) on a Cobas c501 analyzer. The following formula was used to calculate the level of low-density lipoprotein $(\mathrm{LDL})$ cholesterol: $\mathrm{LDL}$ cholesterol $=$ total cholesterol-HDL cholesterol-(TG/5).

Anthropometric measurements (height, weight, and waist/ hip circumference) and blood pressure measurements were performed at the onset of the study. Blood pressure during the first hospitalization was measured 4 times a day and the mean systolic and diastolic value was recorded in the study data.

IR was quantified using the estimated glucose disposal rate (eGDR) (milligrams/kilogram/minute), calculated with the following equation:

$$
\begin{aligned}
\mathrm{eGDR}= & 24.31-(12.22 \times \mathrm{WHR})- \\
& (3.29 \times \mathrm{HTN})-\left(0.57 \times \mathrm{HbA}_{1 \mathrm{c}}\right),
\end{aligned}
$$

where WHR is waist-to-hip ratio, HTN is hypertensive status $(0=$ no; $1=$ yes $)$, and $\mathrm{HbA}_{1 \mathrm{c}}$ is expressed as a percentage. A decrease in eGDR reflects an increase in IR. PE and GH were diagnosed using criteria proposed by the American College of Obstetricians and Gynecologists [10].

Statistical analyses were performed using MedCalc for Windows, version 12.1.3.0 (MedCalc Software, Mariakerke, Belgium). Testing for normality of data distribution was performed using the D'Agostino-Pearson test. The Kruskal-Wallis test was used for intergroup comparisons. The Chi-square test was used for the comparison of categorical variables. Simple logistic regression was used to search for factors associated with PE and GH. For multivariate comparisons, we used multiple logistic regression with stepwise selection. Based on clinical judgment, we included the following confounders into the stepwise models: maternal age, time since diagnosis of diabetes, body mass index (BMI) at onset of the study, parity (multipara vs. primipara), the presence of vasculopathy, and $\mathrm{HbA}_{1 \mathrm{c}}$. Statistical significance was defined as $P<0.05$ (two sided). The presence of vasculopathy was defined as being diagnosed with at least one of the following: retinopathy, nephropathy, ischemic heart disease. Small for gestational age (SGA) was defined as birth weight lower than 10th percentile, and large for gestational age (LGA) was defined as birth weight greater than 90th percentile using age- and sex-specific regional growth charts [11]. Gestational weight gain (GWG) was categorized based on Institute of Medicine (IOM) 2009 guidelines [12].

The Institutional Ethical Committee of Poznan University of Medical Sciences (No. 673/12, June 12, 2012) approved the study protocol. Informed, written consent was obtained from every patient before inclusion into the study. 


\section{Results}

A total of 179 Caucasian women were included in the study. Of those, 14 women were excluded from the analysis due to spontaneous abortions $(N=13)$ and twin pregnancy $(N=1)$, resulting in a total of 165 women included in the final analysis. Of the women included in the final analysis, 16 (9.7\%) developed PE, 8 (4.8\%) were diagnosed with GH, and 141 did not develop either PE or GH.

The characteristics of the study subgroups are shown in Tables 1and 2. Among the 3 study subgroups, there were no differences in gestational age at the time of study inclusion, maternal age, the proportion of women receiving preconception care, BMI, or WHR. There were no differences in the proportion of women with normal BMI, underweight, and obesity. Proportion of overweight was higher in women who developed PE and PIH than in controls. There was a nonsignificant trend for higher weight gain in women who developed PE. Women who developed PE were diagnosed with diabetes earlier and had a longer duration of the disease than women who were normotensive or had GH. There was significant difference in the proportion of primiparae/ multiparae among subgroups. All PE cases were diagnosed in primiparae. Women who developed PE had higher systolic and diastolic blood pressure than controls, but similar to those who developed GH. There was no difference in systolic and diastolic blood pressure between women with $\mathrm{GH}$ and controls. Birth weight of newborns born to mothers with PE was lower than in normotensive and GH subgroups; however, the proportions of newborns small for gestational age and sex and large for gestational age and sex were comparable between subgroups.

The results of univariate logistic regression are shown in Table 3.

The presence of vasculopathy was the strongest determinant of PE, followed by a history of chronic hypertension and the duration of diabetes. However, chronic hypertension and duration of diabetes were no longer associated with PE after adjustment for the presence of vasculopathy. Higher gestational weight gain (GWG) was associated with $\mathrm{PE}$, and this association remained significant after adjustment for first trimester BMI. Both systolic and diastolic

Table 1 Characteristics of the study group

\begin{tabular}{|c|c|c|c|c|}
\hline & $\begin{array}{l}\text { Control } \\
\text { (a) }\end{array}$ & $\begin{array}{l}\text { GH } \\
\text { (b) }\end{array}$ & $\begin{array}{l}\text { Preeclampsia } \\
\text { (c) }\end{array}$ & $P$ \\
\hline & $N=141$ & $N=8$ & $N=16$ & \\
\hline Gestational age at onset of study, weeks (mean \pm SD) & $9 \pm 2$ & $10 \pm 2$ & $8 \pm 2$ & 0.08 \\
\hline Maternal age at onset of study, years (mean $\pm \mathrm{SD})$ & $29 \pm 4$ & $29 \pm 7$ & $27 \pm 4$ & 0.182 \\
\hline Preconception care $(n[\%])$ & $57(40.4)$ & $3(37.5)$ & $3(18.8)$ & 0.2842 \\
\hline Age at the time of diagnosis of diabetes $($ mean $\pm \mathrm{SD})$ & $17 \pm 8 \mathrm{c}$ & $21 \pm 8 c$ & $10 \pm 4 \mathrm{a}, \mathrm{b}$ & 0.001 \\
\hline Duration of diabetes, years (mean $\pm \mathrm{SD}$ ) & $12 \pm 7 \mathrm{c}$ & $12 \pm 8 \mathrm{c}$ & $17 \pm 7 \mathrm{a}, \mathrm{b}$ & 0.0196 \\
\hline BMI at onset of study, $\mathrm{kg} / \mathrm{m}^{2}$ (median [IQR]) & $22.6(20.7-24.8)$ & $24.1(23.1-26.6)$ & $24.7(21.2-26.8)$ & 0.064 \\
\hline Weight distribution $(N ; \%)$ : normal weight (BMI 18.5-24.9) & $100 / 141 ; 70.9 \%$ & $5 / 8 ; 62.5 \%$ & $8 / 16 ; 50 \%$ & 0.15 \\
\hline Underweight $(\mathrm{BMI}<18.5)$ & $6 / 141 ; 4.3 \%$ & $0 / 8 ; 0 \%$ & $1 / 16 ; 6.3 \%$ & 0.15 \\
\hline Overweight (BMI 25.0-29.9) & $26 / 141 ; 18.4 \%$ & $3 / 8 ; 37.5 \%$ & $6 / 16 ; 37.5 \%$ & 0.04 \\
\hline Obese $(\mathrm{BMI}>30)$ & $9 / 141 ; 6.4 \%$ & $0 / 8 ; 0 \%$ & $1 / 16 ; 6.3 \%$ & 0.55 \\
\hline Waist-to-hip ratio at onset of study (mean \pm SD) & $0.79 \pm 0.06$ & $0.80 \pm 0.07$ & $0.76 \pm 0.05$ & 0.282 \\
\hline Gestational weight gain, kg (mean \pm SD) & $11.7 \pm 4.7$ & $12.6 \pm 5.3$ & $14.7 \pm 5.8$ & 0.07 \\
\hline \multicolumn{5}{|c|}{ Gestational weight gain according to Institute of Medicine Guidelines $(N ; \%)$ [12] } \\
\hline Normal & $59 ; 41.8 \%$ & $2 ; 25 \%$ & $6 ; 37.5 \%$ & 0.96 \\
\hline Lower than recommended & $49 ; 34.8 \%$ & $3 ; 37.5 \%$ & $3 ; 18.8 \%$ & 0.60 \\
\hline Higher than recommended & $33 ; 23.4 \%$ & $3 ; 37.5 \%$ & $7 ; 43.8 \%$ & 0.09 \\
\hline Primipara/multipara & $80 / 61$ & $5 / 3$ & $16 / 0$ & 0.0034 \\
\hline Vasculopathy (cases/group, \%) & $28 / 141,19.9 \%$ & $1 / 8,12.5 \%$ & $12 / 16,75 \%$ & 0.05 \\
\hline Systolic blood pressure at onset of the study, $\mathrm{mmHg}($ mean $\pm \mathrm{SD})$ & $115 \pm 11 \mathrm{c}$ & $120 \pm 7$ & $122 \pm 15 \mathrm{a}$ & 0.026 \\
\hline Diastolic blood pressure at onset of the study, $\mathrm{mmHg}($ mean $\pm \mathrm{SD})$ & $71 \pm 8 c$ & $68 \pm 23$ & $76 \pm 9 \mathrm{a}$ & 0.04 \\
\hline Gestational age at delivery, weeks (median [IQR]) & $38(37-39)$ & $38(37-39)$ & $37(36-38)$ & 0.08 \\
\hline Birth weight, g (median [IQR]) & $3540(3120-3900) \mathrm{c}$ & $3780(3510-4205) \mathrm{c}$ & $3040(2833-3615$ a,b & 0.048 \\
\hline SGA newborns (cases/group, \%) & $5 / 141,3.5 \%$ & $0 / 80 \%$ & $0 / 16,0 \%$ & 0.35 \\
\hline LGA newborns (cases/group, \%) & $44 / 141,31.2 \%$ & $4 / 8,50 \%$ & $5 / 16,31.2 \%$ & 0.31 \\
\hline
\end{tabular}

Letters a,b,c indicate statistical differences between subgroups (Kruskal-Wallis test) 
Table 2 Selected laboratory parameters

\begin{tabular}{|c|c|c|c|c|}
\hline & $\begin{array}{l}\text { Control } \\
\text { (a) } \\
N=141\end{array}$ & $\begin{array}{l}\mathrm{GH} \\
\text { (b) } \\
N=8\end{array}$ & $\begin{array}{l}\text { Preeclampsia } \\
\text { (c) } \\
N=16\end{array}$ & $P$ \\
\hline $\mathrm{HbA}_{1 \mathrm{c}} \mathrm{I} ; \% ; \mathrm{mmol} / \mathrm{mol}$ & $\begin{array}{l}6.6(5.9-7.5) \mathrm{c} \\
49(41-58)\end{array}$ & $\begin{array}{l}6.6(6.3-6.9) \\
49(45-52)\end{array}$ & $\begin{array}{l}7.8(6.6-8.9) \mathrm{a} \\
62(49-7.4)\end{array}$ & 0.048 \\
\hline $\mathrm{HbA}_{1 \mathrm{c}} \mathrm{II} ; \% ; \mathrm{mmol} / \mathrm{mol}$ & $\begin{array}{l}5.6(5.2-6.0) \mathrm{c} \\
38(33-42)\end{array}$ & $\begin{array}{l}6.0(5.9-6.4) \\
42(41-46)\end{array}$ & $\begin{array}{l}6.1(5.6-6.9) \mathrm{a} \\
43(38-52)\end{array}$ & 0.009 \\
\hline $\mathrm{HbA}_{1 \mathrm{c}} \mathrm{III} ; \% ; \mathrm{mmol} / \mathrm{mol}$ & $\begin{array}{l}5.8(5.5-6.3) c \\
40(37-45)\end{array}$ & $\begin{array}{l}6.2(5.8-6.7) \\
44(40-50)\end{array}$ & $\begin{array}{l}6.3(6.0-7.0) \\
45(42-53)\end{array}$ & 0.004 \\
\hline $\mathrm{eGDR} ; \mathrm{mg} / \mathrm{kg} / \mathrm{min}$ & $10.8(9.6-11.5) \mathrm{c}$ & $11.4(10.8-11.80 \mathrm{c}$ & $10.1(6.6-11.0) \mathrm{a}, \mathrm{b}$ & 0.02 \\
\hline TG I; mmol/L & $0.65(0.52-0.83)$ & $0.68(0.56-0.77)$ & $0.96(0.58-1.17)$ & 0.1 \\
\hline TG II; mmol/L & $1.43(1.16-1.18)$ & $1.37(0.96-1.57)$ & $1.65(1.28-2.28)$ & 0.23 \\
\hline TG III; mmol/L & $2.53(2.03-3.09) \mathrm{c}$ & $2.31(1.91-2.65) \mathrm{c}$ & $3.19(2.55-4.92) \mathrm{a}, \mathrm{b}$ & 0.017 \\
\hline Creatinine clearance $\mathrm{I} ; \mathrm{ml} / \mathrm{min}$ & $131 \pm 35 \mathrm{c}$ & $143 \pm 31 \mathrm{c}$ & $109 \pm 39 \mathrm{a}, \mathrm{b}$ & 0.04 \\
\hline Daily urinary protein excretion; $\mathrm{g} / 24 \mathrm{~h}$ & $0.19(0.09-0.2)$ & $0.15(0.1-0.27)$ & $0.18(0.08-0.22)$ & 0.71 \\
\hline
\end{tabular}

Letters a,b,c indicate statistical differences between subgroups (Kruskal-Wallis test)

blood pressure assessed in the first trimester were significant determinants of PE; however, this association was no longer observed after adjustment for the presence of chronic hypertension. $\mathrm{HbA}_{1 \mathrm{c}}$ levels from all 3 trimesters were significantly associated with $\mathrm{PE}$. There was a negative association between eGDR and PE. Among lipids, TG level in all 3 trimesters was positively associated with $\mathrm{PE}$, and this association was independent of $\mathrm{HbA}_{1 \mathrm{c}}$ levels. There was no association between maternal age, BMI, WHR, preconception care, HDL, LDL, total cholesterol, or 24-h urine protein test and PE. We did not find any predictors of $\mathrm{GH}$ in the regression analysis among all analyzed factors.

\section{Discussion}

The aim of our study was to search for factors associated with PE and GH in women with T1DM. All women included in this prospective, observational study were managed in a single obstetric center for women with diabetes. It gave us the possibility to collect a wide range of clinical and laboratory data from the entire duration of pregnancy. We showed that many factors may be involved in the pathogenesis of PE in women with T1DM; however, their individual predictive value is relatively low. Interestingly, GH had no predictors in our study group. This might be at least partly explained by the small number of patients in the GH subgroup which could have interfered with statistical power of the analysis. However, these discrepancies may also reflect the different pathogenesis of GH and PE. Some authors suggest that GH
Table 3 Univariate analysis of factors predictive for PE in women with T1DM

\begin{tabular}{lcll}
\hline Determinants of PE & Crude OR & $95 \%$ CI & $P$ \\
\hline Duration of diabetes, years & 1.11 & $1.03-1.12$ & 0.009 \\
Vasculopathy (1-yes, 0—no) & 10.84 & $3.27-35.97$ & 0.0001 \\
Chronic hypertension (1-yes, 0—no) & 6.05 & $1.75-20.8$ & 0.004 \\
Gestational weight gain, kg & 1.14 & $1.02-1.28$ & 0.02 \\
Systolic blood pressure at onset of the study, mmHg & 1.06 & $1.01-1.11$ & 0.02 \\
Diastolic blood pressure at onset of the study, mmHg & 1.09 & $1.02-1.16$ & 0.01 \\
HbA1c I; \% & 1.38 & $1.01-1.87$ & 0.04 \\
HbA1c II; $\%$ & 2.76 & $1.43-5.31$ & 0.02 \\
HbA1c III; \% & 2.42 & $1.30-4.51$ & 0.005 \\
eGDR, mg/kg/min & 0.66 & $0.50-0.87$ & 0.003 \\
Triglycerides I; mol/L & 5.32 & $1.65-17.18$ & 0.005 \\
Triglycerides II; mmol/L & 2.52 & $1.02-6.26$ & 0.05 \\
Triglycerides III; mmol/L & 2.28 & $1.39-3.74$ & 0.001 \\
\hline
\end{tabular}


and PE are independent diseases. In their large epidemiological study, Ros et al. [13] demonstrated that T1DM was a significant risk factor for PE, but not for GH. In our study, the incidence of PE was 2 times higher than $\mathrm{GH}$, which seems to confirm the results presented by Ros et al.

Valuable data supporting differences in GH and PE etiology come from studies on maternal hemodynamics and angiogenic biomarkers. Noori et al. [14] demonstrated that women with GH had hyperdynamic circulation and an angiogenic biomarker profile (placental growth factor [PLGF] and soluble fms-like tyrosine kinase-1 [sFlt-1]) similar to normotensive pregnant controls, while these factors were significantly altered in women with PE. Moreover, mothers with $\mathrm{PE}$ (but not with GH) showed venous hemodynamic abnormalities [15]. It has been shown that diabetes, especially when longstanding and complicated by vascular disease, can lead to placental dysfunction, similar to that observed in PE and manifested by changes in both angiogenic biomarkers and restricted fetal growth [16]. In the present study, longer diabetes duration and diabetic vascular disease were associated with PE but not GH. However, diabetes duration was no longer associated with PE after adjustment for the presence of vasculopathy. This indicates that excellent glycemic control as a main method of prevention of diabetic vascular disease can also prevent women from developing PE and its associated complications. Chronic hypertension has been found to predispose to PE [17]. We also found this association in our study; however, it was no longer observed after adjustment for vasculopathy. The effect of hypertension on PE risk might be time dependent, as shown by Sibai et al. In their large, multicenter study, the authors demonstrated that only a history of hypertension lasting for at least 4 years was associated with a higher rate of PE. Due to a relatively small number of patients, we were not able to address such effects in our population. Nonetheless, in a population of T1DM patients, the incidence of hypertension is strongly associated with the presence of diabetic nephropathy, which usually develops many years after the onset of diabetes [18]. Moreover, in the same study, the incidence of hypertension among diabetics with normal urinary protein excretion was similar to that observed in the general population. This suggests that hypertension in this population is rather the consequence of kidney disease. Based on our results, we can also conclude that it is vasculopathy and not hypertension itself that is probably responsible for placental dysfunction and subsequent PE. Intensified antihypertensive treatment in women with diabetic nephropathy, together with aspirin, could probably reduce the rates of PE in this high-risk population [9].

Interestingly, there was no association between increased BMI in the first trimester and PE in our population. Such an association was demonstrated in previous studies and confirmed in a recent meta-analysis [19]. The lack of association between BMI and PE in our study might be explained by the small number of women with overweight and obesity. In a recent population-based cohort study among diabetic women, Persson et al. [20] demonstrated that risk of PE was independent of BMI, but the odds for PE increased further with maternal overweight and obesity among women with T1DM. Nonetheless, the effect of maternal weight on PE risk was rather weak in women with T1DM. According to the authors, this might be explained by the fact that T1DM is a much stronger risk factor for PE than BMI.

Additionally, GWG up to 18 weeks of pregnancy, independent of prepregnancy weight, has been found to increase risk of hypertensive disorders of pregnancy in a general population [21]. In our study, we analyzed GWG over the entire course of pregnancy, but we showed similar results. This suggests that weight control during pregnancy in women with T1DM may also have beneficial effects on the incidence of PE.

Primiparity is a major risk factor for PE. In our study, all PE cases were diagnosed in primiparae. Such disproportion was not observed among controls or the GH subgroup where parity status was comparable. This suggests that primiparity also seems to be a risk factor for PE among women with T1DM; however, such an association was not observed in some studies [22, 23].

Excellent glycemic control is crucial for normal fetal development during pregnancy. We showed that $\mathrm{HbA}_{1 \mathrm{c}}$ levels in all trimesters were positively related to the odds of developing PE, which is in line with the results of previous studies [4, 23-25]. Similarly to the study published by Temple et al., there was no effect of attending preconception care and the incidence of PE in our cohort [4]. However, we found an association between first trimester $\mathrm{HbA}_{1 \mathrm{c}}$ and $\mathrm{PE}$ risk, which is in line with the results of the majority of studies [23-25], but not with the results of Temple et al., who did not show such an association. Bearing in mind the pathogenesis of PE, which is believed to have its roots early in pregnancy, hyperglycemia during that period can negatively impact placental structure and function [26, 27]. Therefore, we believe that strict glycemic control starting from the very first week of pregnancy should be considered for primary prevention of PE in women with T1DM.

Increased IR, other parameter representing oxidative stress has been shown to predispose non-diabetic women and those with gestational diabetes to PE [28]. To the authors' knowledge, this is the first study that evaluated IR in early pregnancy in women with T1DM. For this purpose, we used the eGDR formula - a noninvasive method of IR quantification standardized for patients with T1DM which closely correlates with the gold standard method (glucose clamp technique). eGDR values are inversely correlated with IR. We demonstrated that eGDR was negatively associated with PE. The other novel finding of our study is the positive association between TG concentration in all 3 trimesters and PE. 
A similar association was shown in previous studies; however, it was carried out mostly among non-diabetics [29]. Increased IR and hypertriglyceridemia are typical features of metabolic syndrome, and there is a need for interventional trials to determine whether prepregnancy weight reduction and dietary intervention could lower the risk of PE in women with T1DM.

PE has been shown to predispose to intrauterine growth restriction. Interestingly there were no newborns with SGA in both PE and PIH groups. For the purpose of this study, we used percentile charts for general population. Pearson et al. demonstrated that birth weight distribution of offspring born to women with type 1 diabetes is shifted to the right of the normal reference [30]. It may be hypothesized that some growth-restricted fetuses of the diabetic mothers can be falsely "normalized" by maternal hyperglycemia. This could have been possible in our population of women with PE as their metabolic control was worse than in controls.

Our study has limitations. Like in other prospective studies, the number of women who developed PE and GH was small. Moreover, we did not include data on aspirin use in the analysis. However, until 2015, there were no recommendations for such prophylaxis in women with pregestational diabetes in Poland; therefore, it was very rarely used by pregnant women at the time of the study.

The strength of the current study is that all women were managed in a single center according to the same protocols. Because all women with T1DM admitted to our department during the study period were recruited to the study and we used a nested case-control design, selection bias was minimized. Furthermore, the study period was relatively short, and we used comparable therapeutic targets throughout the study. Moreover, all laboratory measurements were performed in the same certified laboratory, thus limiting the possibility of non-random errors resulting from procedural differences. Finally, because of the prospective design, we were able to collect a wide range of clinical and laboratory data including estimation of eGDR, which is novel in the literature.

To summarize, we demonstrated that the incidence of PE among women with T1DM remains high and many factors might be involved in its pathogenesis. However, there is a need for further research in this area, especially aimed at searching for effective methods for prevention of PE, such as dietary interventions and prepregnancy weight control to reduce IR in early pregnancy.

Acknowledgements We would like to thank our patients for their participation in the study. This work was supported by Poznan University of Medical Sciences (Grant No. 502-14-01110141-99677, to PG) and Polish Diabetes Association (PG is a recipient of the Professor Artur Czyżyk Scientific Grant).

\section{Compliance with ethical standards}

\section{Conflict of interest None.}

Ethical approval All procedures performed in studies involving human participants were in accordance with the ethical standards of the institutional and/or national research committee and with the 1964 Helsinki Declaration and its later amendments or comparable ethical standards. The Institutional Ethical Committee of Poznan University of Medical Sciences (No. 673/12, June 12, 2012)approved the study protocol.

Informed consent Informed consent was obtained from all individual participants included in the study.

Open Access This article is distributed under the terms of the Creative Commons Attribution 4.0 International License (http://creativecommons.org/licenses/by/4.0/), which permits unrestricted use, distribution, and reproduction in any medium, provided you give appropriate credit to the original author(s) and the source, provide a link to the Creative Commons license, and indicate if changes were made.

\section{References}

1. Lisonkova S, Joseph KS (2013) Incidence of preeclampsia: risk factors and outcomes associated with early- versus lateonset disease. Am J Obstet Gynecol 209(6):544. doi:10.1016/j. ajog.2013.08.019

2. Weissgerber TL, Mudd LM (2015) Preeclampsia and diabetes. Curr Diabetes Rep 15(3):579. doi:10.1007/s11892-015-0579-4

3. Wender-Ozegowska E, Kozlik J, Biczysko R, Ozegowski S (2004) Changes of oxidative stress parameters in diabetic pregnancy. Free Radic Res 38(8):795-803

4. Temple RC, Aldridge V, Stanley K, Murphy HR (2006) Glycaemic control throughout pregnancy and risk of pre-eclampsia in women with type I diabetes. BJOG 113(11):1329-1332. doi:10.1111/j.1471-0528.2006.01071.x

5. Spracklen CN, Smith CJ, Saftlas AF, Robinson JG, Ryckman KK (2014) Maternal hyperlipidemia and the risk of preeclampsia: a meta-analysis. Am J Epidemiol 180(4):346-358. doi:10.1093/aje/ kwu 145

6. Srinivas SK, Sammel MD, Bastek J et al (2009) Evaluating the association between all components of the metabolic syndrome and pre-eclampsia. J Matern Fetal Neonatal Med 22(6):501-509. doi:10.1080/14767050902794642

7. Gutaj P, Wender-Ożegowska E, Brązert J (2016) Maternal lipids associated with large-for-gestational-age birth weight in women with type 1 diabetes: results from a prospective single-center study. Arch Med Sci. doi:10.5114/aoms.2016.58619

8. Gutaj P, Krzyzanowska P, Brazert J, Wender-Ozegowska E (2016) Determinants of C-reactive protein concentrations in pregnant women with type 1 diabetes. Pol Arch Med Wewn 126(4):230-236

9. Nielsen LR, Damm P, Mathiesen ER (2009) Improved pregnancy outcome in type 1 diabetic women with microalbuminuria or diabetic nephropathy: effect of intensified antihypertensive therapy? Diabetes Care 32(1):38-44. doi:10.2337/dc08-1526

10. ACOG (2013) Hypertension in pregnancy. Report of the American College of Obstetricians and Gynecologists' task force on hypertension in pregnancy. Obstet Gynecol 122(5):1122-1131. doi:10.1097/01.AOG.0000437382.03963.88 
11. Gadzinowski J, Kaliszewska-Drozdowska MD, Kosinska M, Mazela J, Stoinska B (2003) Birth weight and gestational age of newborns from Wielkopolski and Lubuski regions. Ginekol Pol 74(3):186-192

12. IOM (2009) Weight gain during pregnancy: reexamining the guidelines. National Academies Press (US). doi:10.17226/12584

13. Ros HS, Cnattingius S, Lipworth L (1998) Comparison of risk factors for preeclampsia and gestational hypertension in a populationbased cohort study. Am J Epidemiol 147(11):1062-1070

14. Noori M, Donald AE, Angelakopoulou A, Hingorani AD, Williams DJ (2010) Prospective study of placental angiogenic factors and maternal vascular function before and after preeclampsia and gestational hypertension. Circulation 122(5):478-487. doi:10.1161/CIRCULATIONAHA.109.895458

15. Gyselaers W, Staelens A, Mesens T et al (2015) Maternal venous Doppler characteristics are abnormal in pre-eclampsia but not in gestational hypertension. Ultrasound Obstet Gynecol 45(4):421426. doi:10.1002/uog. 13427

16. Gutaj P, Wender-Ozegowska E, Iciek R, Zawiejska A, Pietryga M, Brazert J (2014) Maternal serum placental growth factor and fetal SGA in pregnancy complicated by type 1 diabetes mellitus. J Perinat Med 42(5):629-633. doi:10.1515/jpm-2013-0227

17. Sibai BM, Lindheimer M, Hauth J et al (1998) Risk factors for preeclampsia, abruptio placentae, and adverse neonatal outcomes among women with chronic hypertension. N Engl J Med 339(10):667-671. doi:10.1056/nejm199809033391004

18. Norgaard K, Feldt-Rasmussen B, Borch-Johnsen K, Saelan H, Deckert T (1990) Prevalence of hypertension in Type 1 (insulin-dependent) diabetes mellitus. Diabetologia 33(7):407-410. doi:10.1007/bf00404089

19. Bartsch E, Medcalf KE, Park AL, Ray JG (2016) Clinical risk factors for pre-eclampsia determined in early pregnancy: systematic review and meta-analysis of large cohort studies. BMJ. doi:10.1136/bmj.i1753

20. Persson M, Cnattingius S, Wikstrom A-K, Johansson S (2016) Maternal overweight and obesity and risk of pre-eclampsia in women with type 1 diabetes or type 2 diabetes. Diabetologia 59(10):2099-2105. doi:10.1007/s00125-016-4035-z

21. Macdonald-Wallis C, Tilling K, Fraser A, Nelson SM, Lawlor DA (2013) Gestational weight gain as a risk factor for hypertensive disorders of pregnancy. Am J Obstet Gynecol 209(4):327-e1. doi:10.1016/j.ajog.2013.05.042

22. Funai EF, Paltiel OB, Malaspina D, Friedlander Y, Deutsch L, Harlap S (2005) Risk factors for pre-eclampsia in nulliparous and parous women: the Jerusalem perinatal study. Paediatr Perinat Epidemiol 19(1):59-68. doi:10.1111/j.1365-3016.2004.00623.x

23. Castiglioni MT, Valsecchi L, Cavoretto Pet al (2014) The risk of preeclampsia beyond the first pregnancy among women with type 1 diabetes parity and preeclampsia in type 1 diabetes. Pregnancy Hypertens 4(1):34-40. doi:10.1016/j.preghy.2013.09.001

24. Hanson U, Persson B (1998) Epidemiology of pregnancy-induced hypertension and preeclampsia in type 1 (insulin-dependent) diabetic pregnancies in Sweden. Acta Obstet Gynecol Scand 77(6):620-624

25. Hsu CD, Hong SF, Nickless NA, Copel JA (1998) Glycosylated hemoglobin in insulin-dependent diabetes mellitus related to preeclampsia. Am J Perinatol 15(3):199-202. doi:10.105 5/s-2007-993926

26. Weiss U, Cervar M, Puerstner P et al (2001) Hyperglycaemia in vitro alters the proliferation and mitochondrial activity of the choriocarcinoma cell lines BeWo, JAR and JEG-3 as models for human first-trimester trophoblast. Diabetologia 44(2):209-219. doi:10.1007/s001250051601

27. Hiden U, Glitzner E, Ivanisevic M et al (2008) MT1-MMP expression in first-trimester placental tissue is upregulated in type 1 diabetes as a result of elevated insulin and tumor necrosis factoralpha levels. Diabetes 57(1):150-157. doi:10.2337/db07-0903

28. Gutaj P, Sawicka-Gutaj N, Brazert M, Wender-Ozegowska E (2015) Insulin resistance in pregnancy complicated by type 1 diabetes mellitus. Do we know enough? Ginekol Pol 86(3):219-223

29. Ray JG, Diamond P, Singh G, Bell CM (2006) Brief overview of maternal triglycerides as a risk factor for pre-eclampsia. BJOG 113(4):379-386. doi:10.1111/j.1471-0528.2006.00889.x

30. Persson M, Pasupathy D, Hanson U, Norman M (2011) Birth size distribution in 3705 infants born to mothers with type 1 diabetes: a population-based study. Diabetes Care 34(5):1145-1149. doi: $10.2337 / \mathrm{dc} 10-2406$ 